\title{
Why so many unknown genes? Partitioning orphans from a representative transcriptome of the lone star tick Amblyomma americanum
}

\author{
Amanda K Gibson ${ }^{1 *}$, Zach Smith², Clay Fuqua ${ }^{1}$, Keith Clay ${ }^{1}$ and John K Colbourne 2,3
}

\begin{abstract}
Background: Genomic resources within the phylum Arthropoda are largely limited to the true insects but are beginning to include unexplored subphyla, such as the Crustacea and Chelicerata. Investigations of these understudied taxa uncover high frequencies of orphan genes, which lack detectable sequence homology to genes in pre-existing databases. The ticks (Acari: Chelicerata) are one such understudied taxon for which genomic resources are urgently needed. Ticks are obligate blood-feeders that vector major diseases of humans, domesticated animals, and wildlife. In analyzing a transcriptome of the lone star tick Amblyomma americanum, one of the most abundant disease vectors in the United States, we find a high representation of unannotated sequences. We apply a general framework for quantifying the origin and true representation of unannotated sequences in a dataset and for evaluating the biological significance of orphan genes.

Results: Expressed sequence tags (ESTs) were derived from different life stages and populations of $A$. americanum and combined with ESTs available from GenBank to produce 14,310 ESTs, over twice the number previously available. The vast majority (71\%) has no sequence homology to proteins archived in UniProtKB. We show that poor sequence or assembly quality is not a major contributor to this high representation by orphan genes. Moreover, most unannotated sequences are functional: a microarray experiment demonstrates that $59 \%$ of functional ESTs are unannotated. Lastly, we attempt to further annotate our EST dataset using genomic datasets from other members of the Acari, including Ixodes scapularis, four other tick species and the mite Tetranychus urticae. We find low homology with these species, consistent with significant divergence within this subclass.

Conclusions: We conclude that the abundance of orphan genes in A. americanum likely results from 1) taxonomic isolation stemming from divergence within the tick lineage and limited genomic resources for ticks and 2) lineagespecific genes needing functional genomic studies to evaluate their association with the unique biology of ticks. The EST sequences described here will contribute substantially to the development of tick genomics. Moreover, the framework provided for the evaluation of orphan genes can guide analyses of future transcriptome sequencing projects.
\end{abstract}

Keywords: Amblyomma americanum, Chelicerata, EST (Expressed Sequence Tags), Microarray, Orphan genes, Taxonomic isolation, Tick, Transcriptome

\footnotetext{
* Correspondence: amakgibs@indiana.edu

'Department of Biology, Indiana University, Bloomington, IN 47405, USA

Full list of author information is available at the end of the article
} 


\section{Background}

Genome sequencing efforts focused upon the phylum Arthropoda have grown enormously with advances in genomics and bioinformatics. As of May, 2012, the National Center for Biotechnology Information (NCBI) reported 222 arthropod genomes as assembled or in progress. Importantly, $82 \%$ of these projects are for true insects (Hexapoda: Insecta). Genomic resources for the remainder of the arthropod phylum are far more limited: 24 projects are reported for crustaceans, one for myriapoda, and 16 for chelicerates. The subphyla have divergent evolutionary histories exceeding 500 million years [1]. Broadening the genome survey across this phylogenetic distance contributes to the discovery of lineage-specific genes [2-4], making the sources of orphan genes a particularly relevant question for these taxa $[5,6]$.

Lack of homology to genomic databases prevents the putative assignment of function to orphans, which typically represent at least $10 \%$ of an organism's gene set $[3,7]$. They are commonly attributed to adaptations associated with a taxon's unique biology [3]. This argument was most recently advocated by researchers of the Daphnia pulex genome, in which a remarkable $36 \%$ of genes showed no homology to other datasets [5]. There are, however, numerous potential sources for orphan gene sequences that must be thoroughly investigated in light of their high representation in sequenced genomes and transcriptomes. Poor quality sequence and/or assembly are the least interesting and arguably most likely sources for unassigned sequences. Moreover, genes without homology to other datasets may be non-functional [3,8-13]. Taxonomic isolation among representative lineages in genome databases can also contribute to lack of homology. For instance, as the first crustacean and chelicerate genomes with annotated genomes, the proportion of orphans in D. pulex and in Tetranychus urticae far exceeds that for closely-related but more heavily-sampled insect genomes [5-7].

In consideration of orphan genes, transcriptome projects serve as an important complement to wholegenome sequencing. They provide a more rapid and less expensive approach to obtaining gene sequences. In addition, transcriptome sequencing projects typically focus exclusively upon protein-coding regions. These are translated to amino acid sequences, which are more likely to be conserved [14-16]. Focusing upon conserved sequences favors identification of true orphan genes. Finally, transcriptomes are an effective proxy for estimating gene diversity and sampling orphan genes when other genomic data are limited. This is contingent upon having a sufficient number of expressed sequence tags (ESTs) that are enriched for full-length transcripts, normalized to sample rare mRNA, and sampled from biologically variable pools of RNA to obtain transcripts associated with diverse tissue types and biological processes $[5,15,17,18]$.

The arthropod subphylum Chelicerata includes scorpions, horseshoe crabs, spiders, mites, and ticks. These lineages are more diverse than Crustacea and equally understudied. The chelicerate subclass Acari comprises the tick and mite lineages. Within the Acari, draft genomes of Tetranychus urticae, the two-spotted spider mite [6], and Ixodes scapularis, the black-legged deer tick $[19,20]$ are available, with that of Rhipicephalus microplus, the southern cattle tick, in progress $[21,22]$. The need for more comprehensive genomic and transcriptomic data within the Acari is pressing given that many species are obligate blood-feeders that vector human and animal pathogens, including typhus, Lyme disease, Rocky Mountain Spotted Fever, and ehrlichiosis [23]. More data from blood-feeding chelicerates would allow comparison within ticks and with blood-feeding insects for identification of shared pathways to be exploited in control efforts. Indeed, numerous transcriptome projects targeting the salivary glands of at least 12 tick species have implicated several gene families as central to blood-feeding [24-35].

The lone star tick, Amblyomma americanum, is one of the most abundant vectors of zoonotic pathogens in the United States [36-38]. White-tailed deer are key hosts of A. americanum, and their ongoing expansion into suburban areas has increased tick-human interactions [36-38]. Lone star tick bites are associated with many diseases including human monocytic ehrlichiosis [39], southern tick-associated rash illness [40,41], tularemia [42], several pathogenic Rickettsia [36-38,43], and perhaps the recently discovered Heartland Virus [44]. As of September 2012, only 6,502 ESTs were available for A. americanum on GenBank [45], derived primarily from specific analysis of gene expression associated with tick salivary glands and blood-feeding $[32,46]$. A wholeorganism transcriptome would complement these previously available sequences by increasing gene number and diversity.

Here, we present a comprehensive study of a normalized EST library for A. americanum enriched for unique, non-redundant transcripts. This library more than doubles the number of sequences previously available for this species. It represents a compilation of sequences from five life stages from a laboratory colony (i.e. larva, nymph, adult male, adult female, engorged female) and from a cohort of ticks collected from the wild. This approach reveals a large number of genes lacking homology to existing tick and other arthropod genomic datasets. We also outline a framework for evaluating orphan genes, with the aim to distinguish the primary sources of non-homology. Our results argue for a greater recognition and critical assessment of lineage-specific genes, notably in ticks and other understudied taxa. 


\section{Results/Discussion}

\section{EST sampling and sequencing}

cDNA libraries were constructed from five developmental stages (larvae, nymph, adult male, adult female, engorged female) of Amblyomma americanum, reared under laboratory conditions. An additional library was constructed from a wild-collected population of 50 adult males and 50 adult females. Each library was normalized to reduce redundancy and improve gene discovery, particularly of rare transcripts. Details of preparation and single-pass Sanger-sequencing are provided in the Methods section. The numbers of cloned cDNA inserts derived from each of the libraries are presented in Additional file 1: Table S1.

\section{Assembly}

The ESTPiper analysis tool [47] was applied for base calling and data cleaning, resulting in removal of 4,866 low-quality reads. The CAP3 [48] component of the ESTPiper sequence analysis tool was used to assemble the remaining 15,390 high quality sequences. The assembly yielded 12,319 unique sequences, comprising 10,443 singletons and 1,876 contigs, of which $86 \%$ were assemblies of two to three sequences (Additional file 1: Table S2a). Average redundancy was therefore estimated at $12 \%$, with average gene discovery accordingly estimated at $88 \%$.

An additional 6,502 A. americanum ESTs were available through GenBank. To enhance the size of our dataset, the 12,319 unique sequences generated from our six normalized libraries were secondarily assembled with these GenBank sequences using the CAP3 component of the ESTPiper. This nested assembly procedure allowed a primary, high-quality assembly of our six normalized libraries, followed by a secondary assembly with the potentially more variable and polymorphic sequences from GenBank. In doing so, we aimed to obtain a greater representation of gene transcripts. The secondary assembly produced 14,310 unique sequences, comprising 11,580 singletons, and 2,730 contigs, of which $76 \%$ were assemblies of two to three sequences (Additional file 1: Table S2b). Average redundancy was estimated at $14 \%$ and average gene discovery at $86 \%$. The 6,502 GenBank sequences were therefore on average more likely to be redundant than our initial assembly of the six normalized libraries.

The distribution of ESTs across the six individual libraries (larvae, nymph, adult male, adult female, engorged female, and wild-collected) allowed examination of variation in expressed genes across developmental stage. This analysis served to identify transcripts that are expressed preferentially in a specific life stage or population. The results are discussed in detail in the Additional file 2.

\section{Annotation}

The assembled sequences were processed using the ESTPiper for annotation against the UniProtKB protein database (date: August 7, 2011) [49]. Of the 14,310 sequences, 4,118 (29\%) matched at least one known protein. All BLAST searches reported in this study limit returns to an e-value cutoff of $1 \times 10^{-5}$ and a minimum length of 33 aligned amino acid residues. Among the 2,730 assembled contigs, 1,243 (46\%) matched at least one known protein (Table 1). For all sequences, the distribution of e-value scores indicated that our search against the UniProtKB database provided predominantly strong matches: $86 \%$ had scores $\leq 1 \times 10^{-10}$ and $24 \%$ had scores $\leq 1 \times 10^{-50}$ (Figure $1 \mathrm{~A}$ ). For protein matches against the 1,243 annotated contigs, the distribution of e-value scores demonstrated a similar pattern: $80 \%$ had scores $\leq 1 \times 10^{-10}$ and $32 \%$ had scores $\leq 1 \times 10^{-50}$. Therefore, the number of ESTs in our dataset with a protein match is proportionally low, but the returned matches were overall significant.

For the $29 \%$ of EST sequences matching a known protein, a survey of the distribution of taxonomic

Table 1 Summary of the annotation of the secondary Amblyomma americanum EST assembly

\begin{tabular}{ll}
\hline A. UniProtKB Annotation & Number \\
Input sequences & 14,310 \\
Short (<33 amino acids) returns & 171 \\
Weak (e-value > 1E-5) returns & 145 \\
Total unannotated returns & 10,192 \\
Annotated singletons & 2,875 \\
Annotated contigs & 1,243 \\
Total annotated sequences & 4,118 \\
\hline B. Peptide Database: Species & No. matches \\
Ixodes scapularis & 2,842 \\
Tetranychus urticae & 143 \\
Daphnia pulex & 208 \\
Pediculus humanus & 107 \\
Acyrthosiphon pisum & 225 \\
Apis mellifera & 140 \\
Tribolium castaneum & 218 \\
Bombyx mori & 28 \\
Aedes aegypti & 49 \\
Anopheles gambiae & 39 \\
Culex quinquefasciatus & 46 \\
Drosophila melanogaster & 52 \\
Total Hits & 399 (28.6\%) \\
\hline
\end{tabular}

Statistics given for annotation against (A) the UniProtKB protein database and (B) a collection of arthropod datasets. A BLAST search of $A$. americanum ESTs against a compilation of 12 arthropod peptide datasets was conducted. Species are arranged to reflect increasing phylogenetic distance from $A$. americanum. 


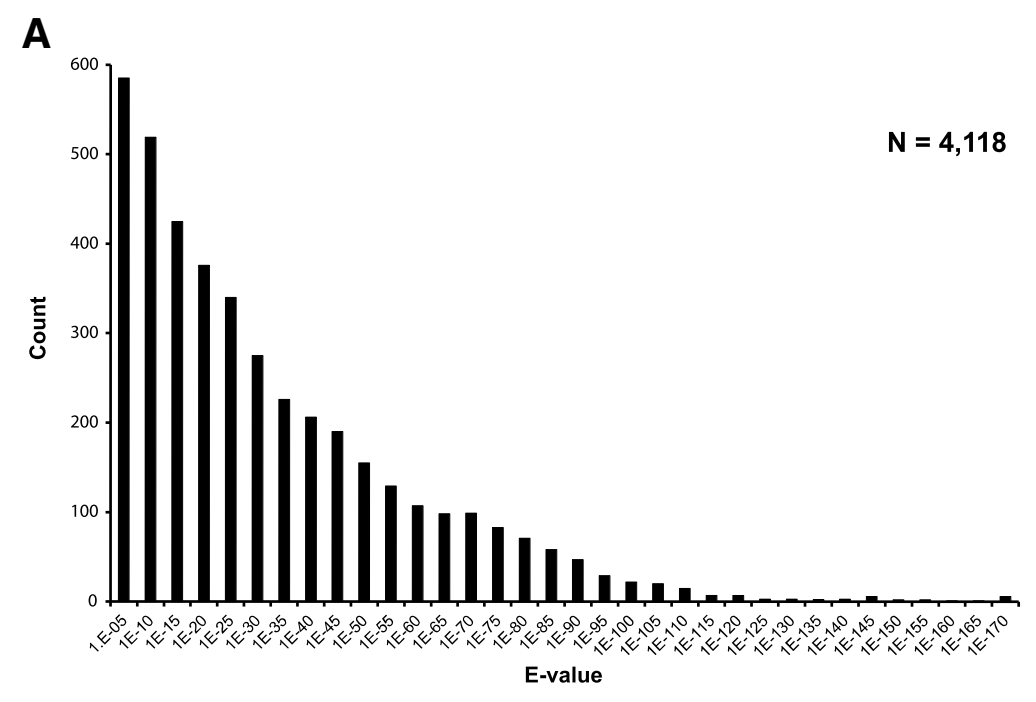

B

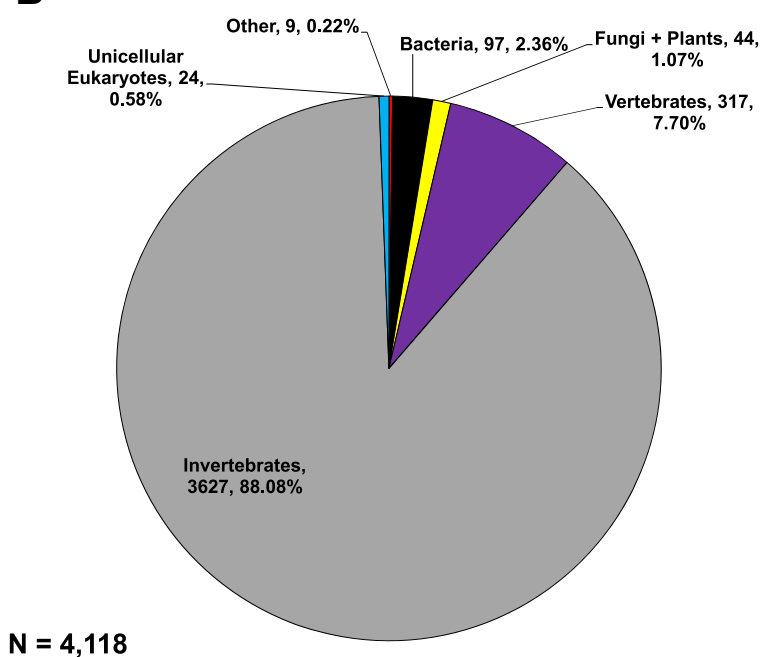

C

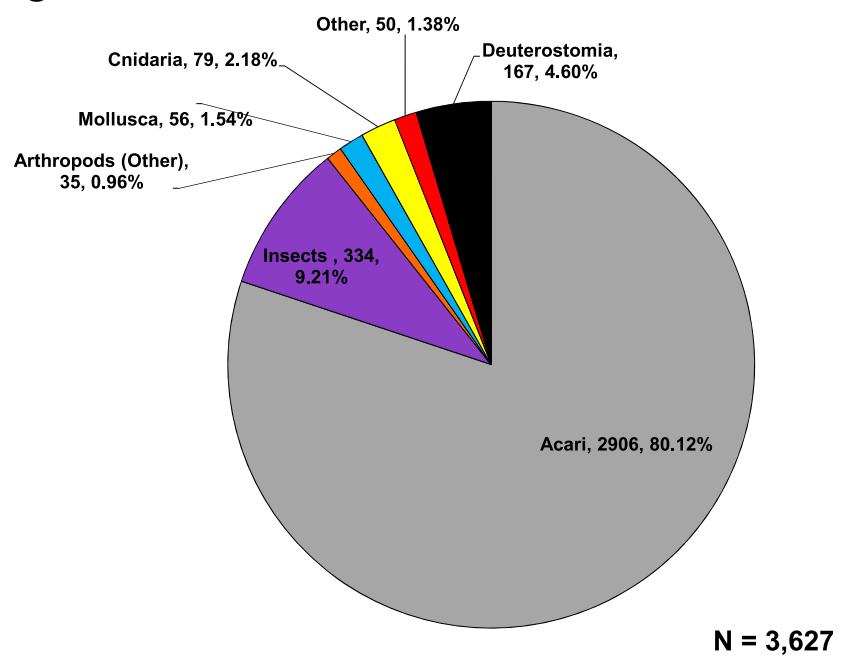

Figure 1 Summary of the UniProtKB annotation of the secondary assembly of the Amblyomma americanum EST library. (A) The e-value distribution of all annotated returns and the taxonomic distribution of (B) all annotated returns $n=4,118$ and (C) returns annotated as invertebrate $\mathrm{n}=3,627$.

domains for returned comparative protein alignments revealed that the vast majority $(88 \%)$ of $A$. americanum sequences matched proteins derived from invertebrates (Figure 1B). More specifically, 71\% matched proteins from Acari lineages, and 9\% matched proteins from other arthropods, predominantly insects (Figure 1C). In total, 4,015 of the annotated returns matched eukaryotic proteins, while six matched proteins derived from viruses and 97 from bacteria. The majority of these bacterial annotations $(\mathrm{n}=76,78 \%)$ were identified as members of the gram-negative $\gamma$-proteobacterial family Coxiellaceae. These sequences likely derive from the Coxiella sp. endosymbiont of $A$. americanum and are discussed in detail in the Additional file 2.
To investigate gene conservation more broadly across the arthropod phylum, we conducted a BLAST search of our A. americanum EST library against the predicted peptides of nine insect, one crustacean, and two chelicerate species with quality genome annotations. Of the 14,310 assembled EST sequences, 4,099 (29\%) matched at least one arthropod peptide (Table 1). This proportion is nearly identical to the proportion of EST sequences matching proteins in UniProtKB. As expected, the vast majority ( $\mathrm{n}=2,842,69 \%)$ of these matches were to I. scapularis peptides. The next most highly represented taxonomic groups were the aphid Acyrthoshipon pisum at 225 matches, the flour beetle Tribolium castaneum at 218 , and the crustacean $D$. pulex at 208. The least represented groups were the three mosquito species 
Aedes aegypti at 49 matches, Culex quinquefasciatus at 46, and Anopheles gambiae at 39, and the silk moth Bombyx mori at 28 (Table 1). Low sequence homology was also observed between $A$. americanum and a fellow member of the Acari, T. urticae, indicating significant diversification within this subclass.

\section{Why are there so many unknown sequences in the Amblyomma americanum transcriptome?}

In the genomic comparisons reported thus far, no more than $29 \%$ of the $A$. americanum transcriptome match genes that were annotated in other organisms. Therefore, at least $71 \%$ of the ESTs lack homology to preexisting datasets based upon this preliminary annotation. We present here four potential sources of these unknown genes and assess their validity as explanations for the high representation observed in the A. americanum transcriptome. Because high proportions of unknown genes are commonly observed when annotating transcriptomes for a wide diversity of taxa [13,50-52], this framework is designed to help guide the reporting of unknown genes in future transcriptome projects.

\section{Hypothesis 1: the sequences are low quality}

Unannotated ESTs may be attributed to differences in sequence quality, as measured by length of predicted open reading frames (ORFs), presence of start codons, EST nucleotide length, and GC-content [3,8-13]. The most striking contrast between unannotated and annotated sequences from our study was ORF length. The mean ORF length, given by OrfPredictor, was significantly shorter for unannotated EST sequences as compared to annotated ESTs $(\mathrm{t}=53.19 ; \mathrm{p}<0.0001$, $\mathrm{df}=5215$ ) (Table 2, Figure 2). Likewise, the average length of annotated contigs was longer than that of unannotated contigs. Additionally, the mean nucleotide length of annotated ESTs was significantly larger

Table 2 Comparison of quality measures for annotated and unannotated ESTs

\begin{tabular}{rlll}
\hline & & Annotated & Unannotated \\
\hline Mean ORF length (nts) & Sequences & 339.9 & 183.4 \\
& Contigs & 376.7 & 213.1 \\
Mean length (nts) & Sequences & 594.9 & 536.0 \\
& Contigs & 746.5 & 735.8 \\
Number w/o start codon & Sequences & 0 & 398 \\
& Contigs & 0 & 38 \\
Mean GC-content & Sequences & 50 & 45.2 \\
& Contigs & 49.7 & 45.1 \\
\hline
\end{tabular}

Comparisons are included for mean open-reading frame (ORF) length, mean nucleotide length, number of ESTs lacking a start codon, and mean GCcontent. Statistics were obtained using OrfPredictor (ORF length and start codons) and Geneious software (nucleotide length and GC-content). than that of unannotated ESTs $(t=15.27 ; \mathrm{p}<0.0001$, $\mathrm{df}=7824$ ). Accordingly, the mean nucleotide length of annotated contigs was longer than that of unannotated contigs. OrfPredictor also did not predict a start codon for $4 \%$ of the 10,192 unannotated sequences and, more specifically, for $3 \%$ of the 1,487 unannotated contigs. Lastly, the mean GC-content was significantly higher for annotated ESTs relative to unannotated ones $(t=41.41$; $\mathrm{p}<0.0001, \mathrm{df}=7917$ ). Likewise, annotated contigs had a higher GC-content than unannotated contigs.

From this analysis of sequence quality, we can reject those sequences without start codons, which represent $4 \%$ of our 10,192 unannotated ESTs. The remaining 9,749 unannotated ESTs represent $70 \%$ of the EST library. Other quality metrics are less conducive to such definitive cut-offs. In spite of their statistical difference, the annotated and unannotated EST sets do not differ dramatically in measured quality scores (Table 2). Even for the most divergent metric, ORF length, the distributions of annotated and unannotated ESTs overlap substantially (Figure 2). Previous studies have consistently shown that sequence quality measures differ between annotated and unannotated sequences, with unannotated sequences shorter on average [3,8-13]. Length disparity was initially interpreted as the result of overassignment of ORFs, with short sequences erroneously assigned as protein-coding regions [11,12]. More recent studies, however, have confirmed that many orphan sequences are indeed protein-coding $[5,10,53,54]$ and that they often encode shorter peptides than annotated sequences $[3,10,55]$. The longer reads of Sanger sequencing and the focus on expressed sequences in this study reduce the risk that unannotated sequences result from overassignment of short ORFs. Functional analysis is required, however, to fully account for the contribution of poor sequence quality to the proportion of unannotated sequences in our dataset (see Hypothesis 3).

\section{Hypothesis 2: the assembly is low quality}

Low assembly quality could contribute to the detection of unannotated sequences if under-assembly occurred and reads lacking homology to other organisms preferentially failed to assemble. Our study benefits from the relatively longer reads of Sanger sequencing. These provide a greater number of potential sequence overlaps during the assembly processes in comparison to the shorter reads of next-generation sequencers. Nevertheless, the rate of assembly failure can be estimated by calculating the percentage of conserved single-copy arthropod genes that are present in multiple copies in the $A$. americanum transcriptome. euGenes/Arthropods [7] reports gene families shared among 14 arthropod families, as well as copy number in each species. Gene family (ARP2) IDs for $A$. americanum ESTs were 


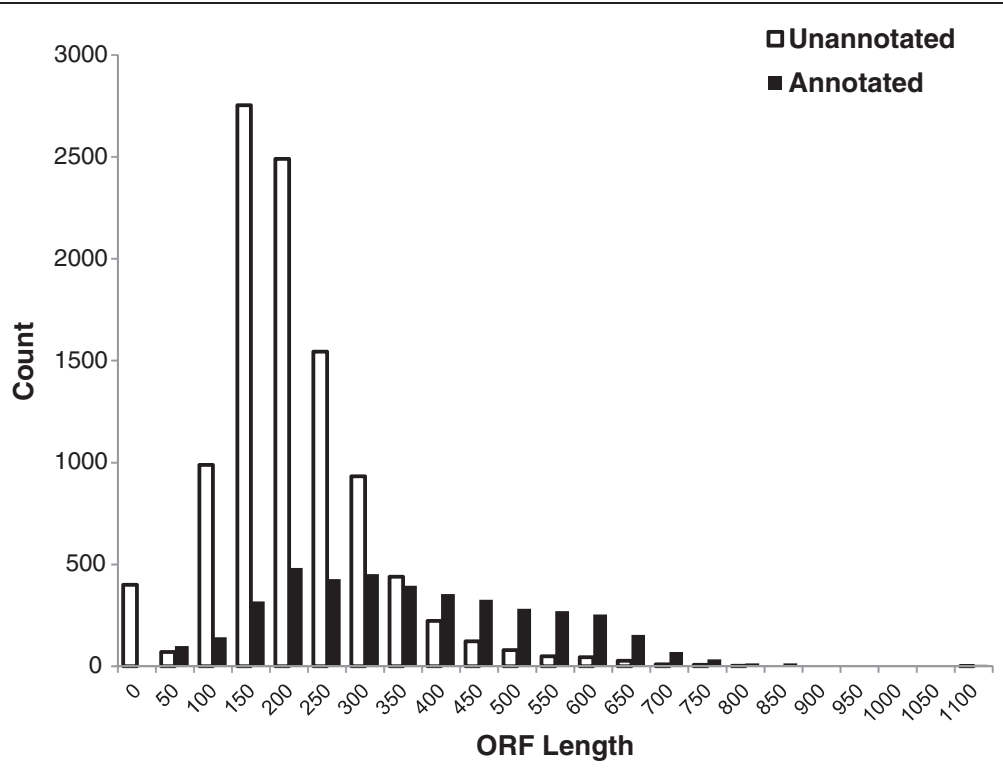

Figure 2 Distribution of the nucleotide lengths of open reading frames for annotated and unannotated EST sequences. Annotated ESTs are shown in black and unannotated in white. Open-reading frame lengths were predicted using the OrfPredictor component of the Transcriptome Analysis Pipeline of the Integrative Services for Genomic Analysis (ISGA) at Indiana University's Center for Genomics and Bioinformatics. Annotation was determined by a BLAST search of the A. americanum EST library against the UniProtKB protein database.

identified through searching for sequence homology against the I. scapularis peptide database. Therefore, the following results only pertain to those ESTs that matched I. scapularis peptides. The ARP2 dataset lists 1,115 gene families that are composed of conserved single-copy orthologs across all 14 arthropod species. Of these, 415 have a match in the A. americanum EST library. A subset of 86 gene families (21\%) contained more than one copy in our A. americanum gene set, likely representing assembly failures rather than duplications in the A. americanum lineage. The majority of these single-copy gene families contain two sequences/contigs in A. americanum, though 12 families had three reported copies, two families had four, and two families had five gene sequences. Therefore, 194 unique sequences in our EST assembly in fact represent only 86 single-copy genes (44\%). The proportion of single-copy gene families present in multiple copies (21\%) multiplied by the proportion of true single-copy genes generated by assembly failures (44\%) suggest an assembly failure rate of $9 \%$. The total number of unique ESTs in our assembly may thereby be reduced by approximately 1,315 ESTs, from 14,310 to 12,995 . This analysis indicates that under-assembly has slightly affected the estimate of the number of non-redundant genes in the A. americanum EST library.

The above analysis alone cannot indicate if underassembly has preferentially failed to assemble unannotated sequences. This might occur, for example, if shorter sequences, which are on average less likely to be annotated, are also less likely to assemble. To specifically address under-assembly of unannotated genes, the assembly process was modified to measure the contribution of assembly quality to the percentage of unannotated sequences. The 12,319 original and 6,502 GenBank ESTs were assembled, without nesting, using Newbler (454 Life Sciences) and updated CAP3 assemblers. Annotation of these two assemblies produced the same results as the previous nested assembly, suggesting that representation of unannotated sequences was not biased by our choice of assembly approach.

We emphasize that our results here do not imply that sequence and assembly quality do not contribute to the detection of unannotated sequences. Rather, we find that, in our dataset, we are unable to detect a strong signal of either low sequence or assembly quality in relation to our estimation of the representation by unannotated sequences.

\section{Hypothesis 3: these sequences do not represent functional genes}

Another explanation for a high proportion of unknown genes is that these sequences do not in fact represent functional genes. Unannotated ESTs may include intronic or untranscribed sequences, due to cloning errors, or sequences with shifted reading frames, due to sequencing errors $[8,56]$. To explore this possibility, we developed microarrays to measure the expression level of 13,962 of the 14,310 A. americanum ESTs across 12 different conditions, distinguished by life stage and environment. A total of 5,000 sequences showed no 
evidence of expression in 11 or 12 conditions by virtue of falling below the detection threshold of a $0.5 \%$ false discovery rate. This threshold was set by the signal level distribution of 11,657 markov-modeled random sequence probes. Therefore, 8,962 (64\%) of ESTs were identified as functional, based upon detectable levels of condition-dependent transcription (Additional file 3: Figure S1). Of these, 3,710 (41\%) matched proteins or sequences from UniProtKB and/or four $I$. scapularis genomic datasets (see Hypothesis 4) (Table 3). This reveals that $41 \%$ of functional $A$. americanum ESTs are annotated, which represents a slight improvement in annotation from the proportions estimated prior to discounting of non-functional ESTs. This is likely a conservative estimate. Many ESTs, in particular unannotated ones [10], may be expressed under limited environmental conditions that are not represented by this microarray experiment, thus leading to their classification as nonfunctional. Importantly, this conservative approach also increases the probability of excluding non-protein-coding transcripts with low or limited expression. These may include processed pseudogenes or mRNA with disrupted reading frames that can contribute to detection of unannotated sequences [56,57]. We conclude from our microarray experiment that there remains in this EST dataset a large proportion of functional genes lacking sequence similarity to genes in annotated genome databases.

\section{Hypothesis 4: taxonomic isolation of A. americanum contributes to detection of unknown sequences}

Ixodes scapularis The availability of a draft genome sequence assembly and annotation for $I$. scapularis allowed investigation of gene conservation across the Metastriata (Amblyomma) and Prostriata (Ixodes) tick groups. These Ixodidae tick lineages are estimated to have shared a most recent common ancestor in the Triassic, approximately 241 mya [58,59]. We predicted

Table 3 Summary of results from microarray validation of functionality of ESTs of Amblyomma americanum

\begin{tabular}{ll}
\hline & Number \\
\hline Total ESTs matching to microarray probes & 13,962 \\
ESTs retained: expression above 0.5\% FDR threshold & 8,962 (64.2\%) \\
Retained ESTs with UniProtKB annotation & 3,105 (34.6\%) \\
Retained ESTs with I. scapularis match & $3,623(40.4 \%)$ \\
Total retained ESTs with annotation & $3,710(41.3 \%)$
\end{tabular}

A subset of ESTs were matched to microarray probes and expression was measured under 12 different conditions. Sequences with expression that fell below a $0.5 \%$ false discovery rate in 11 or 12 of these conditions were rejected. The remaining ESTs were characterized as annotated or unannotated according to matches to the UniProtKB protein database and to four I. scapularis genomic databases. that the percent matching between $A$. americanum ESTs and I. scapularis genomic datasets would be substantially higher than the percent matching to the UniProtKB database, because of shared ancestry of Ixodidae (hard tick) species. Four different datasets were used here to represent the I. scapularis genome: the EST sequences, predicted peptides, assembled contigs, and singletons. Homology of the A. americanum ESTs to these I. scapularis datasets ranged from $16 \%$ to $29 \%$, a surprisingly low percentage that does not differ notably from the percentage of sequences annotated through UniProtKB (Table 4; Additional file 3: Figure S2). Accounting for redundant matches, the four BLAST searches against these I. scapularis datasets returned a total of 5,483 matches, representing $38 \%$ of the A. americanum ESTs. Those sequences with matches to proteins through UniProtKB were far more likely to have a match to I. scapularis: for each of the BLAST searches of A. americanum ESTs against the $I$. scapularis datasets, $73 \%$ or greater of returns also had a match in the UniProtKB database (Table 4). Therefore, the majority of $A$. americanum ESTs was found to have no match to UniProtKB proteins and no homology to I. scapularis genome, peptide, or EST sequences. This result supports a substantial degree of divergence between Ixodes and Amblyomma sequences. Thus unannotated ESTs in the $A$. americanum library may be unique to the Amblyomma or Metastriata lineages of the hard ticks.

Another potential explanation for the low percent matching between $A$. americanum and $I$. scapularis may be the state of the I. scapularis genome. The I. scapularis genome is large $(2,100 \mathrm{Mb})$ and contains a very high proportion of repetitive DNA (70\%) [60,61]. As a result, the current assembly is highly fragmented, with gene regions split across scaffolds. This draft assembly in turn limits the depth of the $I$. scapularis genome annotation [62]. If characteristics of the I. scapularis draft assembly and annotation, such as gene fragmentation, explain the low homology between A. americanum and $I$. scapularis, we predict imperfect matching $(<100 \%)$ of the I. scapularis ESTs to the I. scapularis contigs, singletons, and predicted peptides. We tested this prediction by BLAST searches of the 194,460 I. scapularis ESTs against these three datasets. They returned between 37\% and 66\% matching (Table 4). These values are indeed unexpectedly low for intraspecific BLAST searches, supporting fragmentation and low coverage of the I. scapularis datasets as a potential source of low homology between A. americanum and I. scapularis.

Based upon these results for homology between I. scapularis datasets (Table 4), a proportion of all A. americanum ESTs that failed to match the $I$. scapularis predicted peptides, contigs, and singletons 
Table 4 Summary of BLAST searches of Amblyomma americanum against Ixodes scapularis

\begin{tabular}{|c|c|c|c|c|}
\hline \multirow[b]{2}{*}{ A. Datasets for I. scapularis } & & \multicolumn{2}{|l|}{ UniProtKB } & \multirow{2}{*}{$\begin{array}{l}\text { A. americanum ESTs } \\
\text { Totals }\end{array}$} \\
\hline & & Annotation & No Annotation & \\
\hline \multirow[t]{2}{*}{ ESTs } & Match & 2,997 & 457 & $3,454(24.1 \%)$ \\
\hline & No Match & 1,121 & 9,735 & 10,856 \\
\hline \multirow[t]{2}{*}{ Predicted Peptides } & Match & 3,413 & 179 & $3,592(25.1 \%)$ \\
\hline & No Match & 705 & 10,013 & 10,718 \\
\hline \multirow[t]{2}{*}{ Contigs } & Match & 1,724 & 642 & $2,365(16.5 \%)$ \\
\hline & No Match & 2,394 & 9,550 & 11,944 \\
\hline \multirow[t]{3}{*}{ Singletons } & Match & 3,279 & 845 & $4124(28.8 \%)$ \\
\hline & No Match & 839 & 9,347 & 10,186 \\
\hline & & \multicolumn{2}{|l|}{ I. scapularis ESTs } & \\
\hline \multicolumn{2}{|l|}{ B. Datasets for I. scapularis } & Totals & & \\
\hline \multirow[t]{2}{*}{ Predicted Peptides } & Match & $128,738(66.2 \%)$ & & \\
\hline & No Match & 70,722 & & \\
\hline \multirow[t]{2}{*}{ Contigs } & Match & $72,465(37.3 \%)$ & & \\
\hline & No Match & 121,995 & & \\
\hline \multirow[t]{2}{*}{ Singletons } & Match & $105,183(54.1 \%)$ & & \\
\hline & No Match & 89,277 & & \\
\hline
\end{tabular}

(A) Amblyomma americanum EST sequences matching Ixodes scapularis genomic datasets and (B) I. scapularis EST sequences matching other I. scapularis datasets. Ixodes scapularis datasets include ESTs, predicted peptides, singletons, and assembled contigs. (A) Amblyomma americanum results are categorized according to UniProtKB annotation status (annotation, no annotation) and I. scapularis match status (match, no match). The proportion of the A. americanum ESTs with a match is provided in parentheses for each I. scapularis dataset. (B) BLAST searches of $I$. scapularis ESTs $(\mathrm{N}=194,460)$ against the three other I. scapularis datasets were then conducted to evaluate the quality of these datasets. These are classified according to match status. The proportion of $I$. scapularis ESTs with a match is provided in parentheses.

may be discounted as reflecting characteristics of the I. scapularis reference rather than low homology. If a proportion of the A. americanum ESTs that failed to match the I. scapularis datasets is discarded to control for reference quality, higher values for homology between $A$. americanum and I. scapularis are estimated: 34\% matching between A. americanum ESTs and the I. scapularis predicted peptides, $35 \%$ matching with I. scapularis contigs, and $43 \%$ matching with $I$. scapularis singletons. We therefore conclude that genetic distance of $A$. americanum and I. scapularis, compounded by fragmentation and low coverage of the reference databases of I. scapularis, contributes significantly to the high representation of unknown genes in A. americanum.

\section{Other tick species}

Transcriptomes from additional tick species allowed further investigation of divergence between tick species [24,63-66]. We attempted to investigate if the low homology detected here between $A$. americanum and I. scapularis potentially results from divergence between the Metastriata and Prostriata ticks. Four additional BLAST searches were conducted against the EST libraries of Ixodes ricinus (castor bean tick) (Prostriata), Dermacentor variabilis (American dog tick), Rhipicephalus microplus, and $R$. appendiculatus (brown ear tick)
(Metastriata). EST libraries were obtained from GenBank, and BLAST searches were conducted individually against each of these four databases. The number of matches with A. americanum ESTs was very low: 44 (0.3\%) against I. ricinus, $52(0.4 \%)$ against $D$. variabilis, 270 (1.9\%) against $R$. microplus, and $174(1.2 \%)$ against $R$. appendiculatus. This low percent matching, as compared to I. scapularis, is likely due to the small size of the datasets rather than decreased gene conservation (Additional file 1: Table S3). The size of these datasets precludes any further inference of the degree of divergence between Metastriata and Prostriata ticks. More genomic data for tick species are required to accurately conduct comparative studies [62].

\section{Tetranychus urticae}

In the course of annotating our A. americanum EST dataset, the draft genome sequence of the two-spotted spider mite, Tetranychus urticae, was published [6], making it the only chelicerate and A. americanum's closest relative with a published genome. With the T. urticae genome, we were able to test gene conservation within the subclass Acari, which contains the ticks and the mites. Two datasets represented the $T$. urticae genome: the predicted peptides and the genome scaffold sequences. BLAST searches of the 14,310 A. americanum ESTs returned 2,338 matches (16\%) to the predicted peptides 
and 2,155 (15\%) to the main genome (Additional file 3: Figure S2). As in I. scapularis, the vast majority of these matches were annotated in UniProtKB: $98.2 \%$ for the predicted peptide matches and $97.8 \%$ for the main genome matches. This low homology between Amblyomma and Tetranychus further supports divergence within the Acari as a major source of unknown genes. Indeed, Fukuchi and Nishikawa [13] demonstrate that a lack of close relatives in genomic datasets, as seen with A. americanum, is positively correlated with the proportion of unannotated sequences in a dataset.

\section{What is the biological significance of these unknown genes?}

We demonstrate above that when accounting for sequence quality, assembly quality, and gene function, over $50 \%$ of the ESTs in this $A$. americanum transcriptome lack homology to genomic datasets of other organisms. The majority of these functional, unknown ESTs lack homology to even the relatively closely-related Acari species I. scapularis and T. urticae. These unannotated sequences are thus unique to the lineage leading to Metastriata ticks and, perhaps, more specifically to Amblyomma species. Further genomic resources must be developed for other Prostriata and Metastriata tick species to evaluate the degree of taxonomic restriction of these orphan genes.

The unique ecology and life-history of tick species suggests ample opportunity for lineage-specific adaptations. For example, the blood-feeding habit is the most prominent characteristic of ticks, having been adopted by only a few other arthropod lineages (e.g. mosquitoes and other dipterans, mites, fleas, lice, bedbugs, some hemipterans) [67]. Genomic data are abundant for many blood-feeders that vector diseases, and these data can be used to evaluate the hypothesis that genes associated with the blood-feeding strategy are lineage-specific. The ARP2 dataset includes gene families from five blood-feeding arthropods: A. aegypti, A. gambiae, C. quinquefasciatus, I. scapularis, and the human louse Pediculus humanus. Of the 28,769 ARP2 gene families, $4,428(15 \%)$ are exclusive to blood-feeding arthropods. For thirty random combinations of five species from the 14 composing the ARP2 dataset, the average number of exclusive gene families was 4,639.5 (16\%). The number and proportion of gene families exclusive to bloodfeeders lies well within a single standard deviation of this average and is in fact smaller, indicating that arthropods sharing the blood-feeding habit do not share a greater number of gene families. This suggests that genes associated with blood-feeding may have a high likelihood of being lineage-specific, perhaps due to rapid divergence [10,51] under strong selection exerted by coevolving hosts $[68,69]$. Additionally, of the 4,428 gene families exclusive to blood-feeding arthropods, most gene families $(n=1,753)$ are exclusive to a single species. Only one gene family is shared among all five species and only 23 among four. When three species share a gene family $(n=1,148)$, the species are most commonly the three closely-related mosquitoes.

By BLAST searching against the I. scapularis peptides, 173 of these blood-feeding exclusive gene families were identified in the A. americanum EST library. This is a small fraction (5\%) of the total gene families exclusive to blood-feeding arthropods that were identified in I. scapularis $(\mathrm{n}=3,554)$ (Additional file 1: Table S4). This result corroborates the low homology observed between $A$. americanum and I. scapularis. Moreover, it supports adaptation to blood-feeding as one potential source of unannotated ESTs in this A. americanum transcriptome. We develop the blood-feeding habit here as an example of a trait that defines tick ecology and life-history. A general challenge for ecological and evolutionary genomics is to carry out the necessary functional genetic experiments to evaluate the hypothesis that unannotated sequences are linked to an organism's unique biology.

\section{Conclusions}

Here, we present a characterized set of ESTs for the hard tick A. americanum representing an estimated 14,310 unique sequences. The number of ESTs publicly available for this important North American disease vector is more than doubled by this study. The genomic resources available for $A$. americanum remain limited, however, and our results emphasize the need for an annotated gnome assembly for this species to obtain a more comprehensive representation of its genome. The ESTs we report here will prove a powerful resource in annotation of this future $A$. americanum genome.

Additionally, we reveal up to 5,261 functional genes for which no arthropod or tick homologs are currently available. Using the framework outlined above, only 398 unnanotated ESTs could be definitively eliminated due to poor sequence quality, leaving up to $70 \%(n=9,749)$ of ESTs unannotated. Secondly, our assessment of assembly quality found no evidence for selective amplification of unannotated sequences. Thirdly, we establish that lack of annotation does not arise solely from a lack of gene function. A microarray experiment revealed that $59 \%(\mathrm{n}=5,252)$ of functional ESTs are unannotated. Finally, low homology to I. scapularis and T. urticae, the closest arthropod species with significant genomic resources, showed that taxonomic isolation may contribute significantly to the high representation of unknown genes in the $A$. americanum library. Summarizing across each step of this analysis, our results suggest that the proportion of unannotated, functional genes in this 
A. americanum transcriptome exceeds 50\%. These unannotated sequences may thus represent genes unique to the Amblyomma or Metastriata tick lineages. As taxonomic isolation is reduced by future genome assemblies for $A$. americanum and other hard ticks, the degree of lineage-specific adaptations within tick taxa must be evaluated more closely.

In conclusion, we present a broad overview of $A$. americanum genomics and contribute EST sequences for the development of tick genomics, functional annotation of tick genomic sequences, and enhancement of biological understanding of these major disease vectors. Our findings further confirm that tick lineages are highly divergent [58,59], necessitating whole-genome sequencing of multiple hard tick species. We commend the i5k Insect and other Arthropod Genome Sequencing Initiative. As of January 2013, 8\% of arthropod species nominated for sequencing under this initiative belong to the Chelicerata, the largest representation by any sub-phylum other than the Hexapoda (http://www. arthropodgenomes.org/wiki/i5K). These genomic efforts will both enhance knowledge and facilitate the development of management strategies for tick-borne illnesses. Moreover, we offer a framework for the evaluation of unannotated sequences that can be applied widely, to genomes and transcriptomes of a diversity of taxa. The extension of genomic resources across the tree of life calls for recognition of the significance of unannotated sequences in genomic datasets and for thorough analysis of their biological function.

\section{Methods}

\section{EST sampling and sequencing}

Ticks from five developmental stages (larvae, nymph, adult male, adult female, engorged female) were obtained from the Oklahoma State University Tick Rearing Facility. Additional information regarding these lab-reared colonies can be found at http://www.reeis. usda.gov/web/crisprojectpages/0160810-centralized-tickrearing.html. A natural population of adult ticks (50 males and 50 females) was collected from a field site in Solsberry, Owen County, Indiana, USA. Collections were made in late spring by dragging on a section of private property characterized by a patchwork of old field and young forest vegetation. This group is referred to as "wild-collected."

Total RNA from each sample group (adult male, adult female, engorged female, nymph, larval, wild-collected) was isolated using TRIzol reagent (Invitrogen Life Sciences, Carlsbad, CA) and purified using the RNeasy kit (Qiagen, Valencia, CA). Following removal of DNA and other contaminants with DNAfree (Ambion, Life Sciences, Carlsbad, CA), each RNA sample was quantified with a Nanodrop and qualified with an Agilent Bioanalyzer.
Double-stranded cDNA was constructed and normalized from these six isolated, purified RNA samples using the Trimmer-Direct Normalization Kit (Evrogen, Moscow, Russia) in conjunction with the Creator SMART cDNA Library Construction Kit (Clontech, Mountain View, CA). Normalized libraries were biased towards larger sequences by fractionating the cDNA with CHROMA-SPIN 400 columns (Creator SMART kit) following Sfil digestion. Normalized and digested cDNA was directionally cloned into pDRN-LIB vectors (Clontech). These cDNA inserts were flanked by the Sfil A (5'-GGCCATTACGGCC-3') and Sfil B (5'-GGCCGCCTCGGCC-3') linker sequences. Following ligation, cloned inserts were transformed into TOP10 Electrocomp cells (Invitrogen) via electroporation. Cells were plated onto LB/agar containing $50 \mu \mathrm{g} / \mathrm{mL}$ chloramphenicol and grown overnight at $37^{\circ} \mathrm{C}$.

For each library, a 384-well glycerol stock plate was hand-picked for quality assurance testing. A random subset of 864 clones, representing at minimum, 96 samples from each plate/library, were plasmid-prepped with the PerfectPrep Direct Bind Plasmid Kit (Eppendorf). To determine the sizes of these control samples, each selected cDNA insert was PCR amplified and visualized using agarose gel electrophoresis and a Kodak 440cf imaging station. The average molecular weight of this subset was determined to be 1,185 bp. Normalization efficiency was then assessed by sequencing a single pass 5' read of each cDNA insert using BigDye Terminator ver3.1 sequencing chemistry. Raw trace files were converted using phred2fasta and all reads qualified with TIGR Lucy [70], which trims low quality reads, short reads, vector-only sequences, and mitochondrial reads. Mitochondrial reads were specifically trimmed using the Amblyomma triguttatum mitochondrial genome. A total of 36 inserts were dropped due to sequencer failure, and an additional 32 were excluded due to absence of an insert or short sequence $(<100$ bp). The remaining 796 trimmed reads were assembled with CAP3 [48], yielding 787 contigs and singletons. This initial result corresponds to a gene discovery rate of $99 \%$ among libraries.

After quality assurance, colonies from each of the six libraries were arrayed into 384-well glycerol stock plates and sent to the Genomics Core Facility at Purdue University (Additional file 1: Table S1). Plasmid DNA was amplified from plates using the TempliPhi Rolling Circle Amplification Kit (GE Healthcare, Piscataway, NJ) and sequenced on ABI3730 sequencers using BigDye Terminator ver3.1 sequencing chemistry. The primer pDNRlib30-50fwd (5'-TATACGAAGTTATCAGTCGACG-3') was used for sequencing. The raw trace files were processed with the ESTPiper (http://cas-bioinfo.cas.unt. edu/estpiper/index.html) [47], a web-based analysis tool that processes and assembles ESTs from raw trace files 
through phred2fasta, TIGR Lucy, CAP3, and smaller trimming programs developed by the Center for Genomics and Bioinformatics, Indiana University.

\section{Assembly}

A total of 20,256 cDNA samples were generated from the six normalized $A$. americanum libraries (Additional file 1: Table S2a). Trace files were subjected to base calling with phred and data cleaning through the ESTPiper analysis tool, resulting in removal of 4,866 low-quality sequences. The ESTPiper removed low quality reads, short reads (<100 bp), reads without inserts, vector-only sequences, and mitochondrial sequences. PolyA trimming was applied to only $16 \%$ of sequences during data cleaning, indicating the presence of continuous polyA sequences greater than $15 \mathrm{bp}$. The longest polyA sequence trimmed was $150 \mathrm{bp}$ in length. The remaining 15,390 high quality sequences were submitted to the ESTPiper's CAP3 component for assembly, with overlap match set at $90 \%$. The resulting 12,319 original ESTs were then secondarily assembled, using the ESTPiper's CAP3 assembler and an overlap match of $90 \%$, with the 6,502 ESTs reported by previous studies available at NCBI's GenBank [45] (Additional file 1: Table S2b). Sequences from the six normalized libraries are publicly available through GenBank [dbEST: JZ168803-JZ170971, JZ170972-JZ173026, JZ173027-JZ175168, JZ175169JZ177905, JZ177906-JZ180320, and JZ180321-JZ183760, corresponding to adult female, adult male, engorged female, larval, nymph, and wild-collected transcripts, respectively]. Assembled sequences have been deposited in the Transcriptome Shotgun Assembly database [GenBank TSA: GAGD01000000].

\section{Annotation}

The ESTPiper was used to perform a tBLASTx search of the $A$. americanum EST library against proteins in the UniProtKB database [49] with an e-value threshold of $1 x$ $10^{-5}$. All returns shorter than 33 amino acids were removed. These quality thresholds were applied for all BLAST searches reported in this study.

A BLAST search of the A. americanum EST library was also performed against a combined database of the predicted peptides of nine insect species, Acyrthosiphon pisum (pea aphid), Aedes aegypti (yellow fever mosquito), Anopheles gambiae (African malaria mosquito), Apis mellifera (honey bee), Bombyx mori (silkmoth), Culex quinquefasciatus (Southern house mosquito), Drosophila melanogaster (common fruit fly), Pediculus humanus (human louse), and Tribolium castaneum (red flour beetle), one crustacean, Daphnia pulex (water flea), and two chelicerates, I. scapularis (black-legged deer tick) and the recently sequenced Tetranychus urticae (two-spotted spider mite). These datasets were obtained, respectively, from AphidBase [71], VectorBase (VectorBase [72], http://www.vectorbase.org, A. aegypti Liverpool LVP annotation, Aaegl1), VectorBase ( $A$. gambiae PEST annotation, AgamP3.5), BeeBase [73], SilkDB [74], VectorBase (C. quinquefasciatus Johannesburg annotation, CpipJ1), FlyBase [75], VectorBase ( $P$. humanus USDA annotation, PhumU1), Beetlebase [76], wFleaBase [77], VectorBase (I. scapularis WIKEL annotation), and BOGAS (http://bioinformatics.psb.ugent.be/ webtools/bogas/overview/Tetur, T. urticae).

\section{Framework for investigation of unknown genes Hypothesis 1: sequence quality}

ORFs, ORF lengths, and start codons were predicted for ESTs using the OrfPredictor [78] component of the Transcriptome Analysis Pipeline of the Integrative Services for Genomic Analysis (ISGA) at Indiana University's Center for Genomics and Bioinformatics [79]. Nucleotide lengths and GC-contents were estimated using Geneious [80]. ORF nucleotide lengths, EST nucleotide lengths, and GC-contents of annotated and unannotated ESTs were compared using Welch's T-tests in R v2.12.2.

\section{Hypothesis 2: assembly quality}

ARP2 IDs for arthropod gene families and copy numbers were obtained from eugenes/Arthropods (http://arthropods.eugenes.org/arthropods/) [7]. This database reports gene families shared between 14 arthropod species: 12 insect species (A. pisum, A. aegypti, A. gambiae, A. mellifera, B. mori, C. quinquefasciatus, D. melanogaster, $D$. pseudoobscura, D. mojavensis, Nasonia vitripennis (jewel wasp), P. humanus, and T. castaneum), one crustacean species (D. pulex), and one chelicerate species ( $I$. scapularis). The inclusion of I. scapularis in the ARP2 dataset allowed ARP2 IDs to be assigned to $A$. americanum ESTs. ESTs were first matched to the I. scapularis peptide dataset (see Methods section below, "Phylogenetic distance between A. americanum and other arthropods"). Amblyomma americanum ESTs with a match to I. scapularis were then assigned the ARP2 ID associated with the I. scapularis match. Single-copy ARP2 gene families were extracted by selecting gene families present in all 14 arthropod species with only one copy. The assembly failure rate was calculated by multiplying 1 ) the proportion of single-copy genes that were multi-copy in the A. americanum EST assembly with 2) the proportion of single-copy genes represented by the set of erroneous multi-copy genes in the assembly.

To determine the contribution of assembly quality to the percentage of unannotated sequences, two alternate assemblies were produced by assembling the unassembled 12,319 ESTs reported by this study with GenBank's 6,502 A. americanum ESTs. The two alternate assemblies were produced using Newbler (454 Life Sciences) and the 
updated CAP3 assemblers. These assemblies were then annotated as described previously and contrasted with results for annotation of the original assembly.

\section{Hypothesis 3: functional expression of A. americanum ESTs}

A custom designed microarray was manufactured on the Roche NimbleGen (Madison, WI) multiplex (12-plex) long-oligonucleotide (60 nt) platform. Each glass slide contains 12 identical arrays prepared using a Maskless Array Synthesizer [81]. Each array consists of 137,000 temperature-balanced probes; 13,928 assembled contigs and singletons are represented by nine unique probes, 145 are represented by $<9$ unique probes. The array also contains control probes and 11,657 random probes designed to reflect the genome nucleotide composition by Markov modeling to experimentally determine the appropriate thresholds that measure significant hybridization signals over the background.

RNA from replicated adult, nymph and larval stage animals, both with and without Rickettsia infection, was extracted in TRIzol ${ }^{\circledR}$ following manufacturer's directions (Invitrogen, Carlsbad, CA). The microarray protocol follows previously published methods $[82,83]$. Raw microarray data were processed with the limma package [84] in $R$ version 2.9.0 [85] to normalize expression scores.

To determine if a gene was expressed, we calculated the $99.5 \%$ quantile for expression score of random probes in each individual as the cutoff for calling expression. Thus, for each sample, a called expression is significant at a p-value of 0.005 . For each contig and singleton with more than one probe, we tested the median probe value against this threshold. With this, we determined whether or not a gene had expression support in any of the treatments.

\section{Hypothesis 4: phylogenetic distance between A. americanum and other arthropods}

To determine homology between A. americanum and I. scapularis, several BLAST searches of the $A$. americanum EST library were conducted against various datasets of the I. scapularis genome sequence, which is publicly available through VectorBase. The $I$. scapularis whole-genome sequence assembly and annotation projects are a joint effort of the Broad Institute and the J. Craig Venter Institute. Vectorbase supplies I. scapularis genome sequences $(570,637$ contigs and 7,002,324 unassembled singletons, $\sim 4 \times$ coverage of genome), a library of 194,460 ESTs, and 20,486 predicted peptides, which are a combination of sequences from the I. scapularis EST library and predicted peptides from the available genome sequences. The A. americanum EST library was separately BLASTed against each of these four datasets contigs, singletons, ESTs, and predicted peptides.
These results were then compared to the $A$. americanum BLAST search against the UniProtKB protein database to assess the proportion of $I$. scapularis matches with UniProtKB annotation.

Additionally, BLAST searches of the I. scapularis ESTs against $I$. scapularis genomic datasets (contigs, singletons, and predicted peptides) were conducted to correct for the qualityof the I. scapularis genome assembly. The number of $A$. americanum ESTs that failed to match against an I. scapularis dataset was then multiplied by the proportion of I. scapularis ESTs matching that same dataset, in order to discount the proportion of ESTs that may have failed to match due to fragmentation or low coverage of the I. scapularis genome assembly. After removing these non-matches, the percent matching of the A. americanum EST library against the I. scapularis datasets was re-calculated.

Four additional BLAST searches were conducted against EST datasets for one Prostriata (I. ricinus) and three Metastriata (D. variabilis, $R$. microplus, and $R$. appendiculatus) ticks. All EST datasets were obtained from GenBank,

Finally, BLAST searches of the A. americanum EST library were also conducted against two datasets of the T. urticae genome. Genomic data was produced at the Department of Energy Joint Genome Institute (Walnut Creek, CA, USA). The main genome (640 scaffolds, 89.6 megabases, $\sim 8 \times$ coverage) and the predicted peptides $(18,414)$ of $T$. urticae are available at (http://bioinformatics.psb.ugent.be/webtools/bogas/ overview/Tetur) [6]. Separate BLAST searches of the A. americanum EST library were conducted against the main genome and the predicted peptide datasets as described for the I. scapularis datasets.

\section{Biological significance of unknown genes: blood-feeding} eugenes/Arthropods reports gene families for five species of blood-feeding arthropods: A. aegypti, A. gambiae, C. quinquefasciatus, $P$. humanus, and $I$. scapularis. Gene families found in any of these species and none of the nine non-blood-feeding arthropod taxa were classified as "exclusive" to bloodfeeding taxa. To determine if blood-feeding taxa share a disproportionate number of gene families, the number of gene families exclusive to the five bloodfeeding taxa was compared to the average number of gene families shared by 30 randomly-generated combinations of five of the 14 species included in eugenes/Arthropods. Gene families exclusive to these five blood-feeding arthropods were identified in $A$. americanum using a BLAST search against the $I$. scapularis peptides, as described under Hypotheses 2 and 4. 


\section{Additional files}

Additional file 1: Tables. Includes tables associated with the main text and the Supplemental Text

Additional file 2: Supplemental Text. Includes discussions of the A. americanum EST dataset deconstructed according to life stage, as well as additional annotation results.

Additional file 3: Figures. Figures $\mathrm{S} 1$ through $\mathrm{S} 4$ and associated legends.

\section{Competing interests}

The authors declare that they have no competing interests.

\section{Authors' contributions}

AKG: Assembled and annotated EST library. Performed data analysis, including summary of sequence and assembly quality, comparisons of datasets, and statistical analyses. Participated in design and coordination of study. Drafted the manuscript. ZS: Created the EST library. Contributed to assembly, annotation, and summary of sequence quality. Participated in design and coordination of study. Helped to draft the manuscript. CF: Conceived of the study, participated in its design and coordination, and helped to draft the manuscript. KC: Conceived of the study, participated in its design and coordination, and helped to draft the manuscript. JKC: Participated in assembly, annotation, and data analysis. Performed microarray construction and analysis. Conceived of the study, participated in its design and coordination, and helped to draft the manuscript. All authors read and approved the final manuscript.

\section{Acknowledgements}

We are grateful to Aaron Buechlein of Indiana University's Center for Genomics and Bioinformatics (CGB) for training provided in bioinformatics and his assistance with annotation of the EST dataset. We also thank Doug Rusch of the CGB for helpful discussions and evaluating the quality of the assembly. Jade Carter (from the CGB) and Phillip San Miguel (Purdue University) assisted in the construction of the cDNA library. This work was supported in part by the METACyt Initiative of Indiana University, funded in part through a major grant from the Lilly Endowment, Inc.

\section{Author details}

${ }^{1}$ Department of Biology, Indiana University, Bloomington, IN 47405, USA.

${ }^{2}$ The Center for Genomics and Bioinformatics, Indiana University, Bloomington, IN 47405, USA. ${ }^{3}$ Current address: School of Biosciences, University of Birmingham, Birmingham B15 2TT, United Kingdom.

\section{Received: 24 September 2012 Accepted: 21 February 2013} Published: 27 February 2013

\section{References}

1. Pisani $D$, Poling $L$, Lyons-Weiler $M$, Hedges $S$ : The colonization of land by animals: molecular phylogeny and divergence times among arthropods. BMC Biol 2004, 2(1):1.

2. Kunin V, Cases I, Enright A, de Lorenzo V, Ouzounis C: Myriads of protein families and still counting. Genome Biol 2003, 4:401.

3. Khalturin $\mathrm{K}$, Hemmrich G, Fraune $\mathrm{S}$, Augustin R, Bosch T: More than just orphans: are taxonomically-restricted genes important in evolution? Trends Genet 2009, 25(9):404-413.

4. Wilson GA, Bertrand N, Patel Y, Hughes JB, Feil EJ, Field D: Orphans as taxonomically restricted and ecologically important genes. Microbiology 2005, 151:2499-2501.

5. Colbourne JK, Pfrender ME, Gilbert D, Thomas WK, Tucker A, Oakley TH, Tokishita S, Aerts A, Arnold GJ, Basu MK, et al: The ecoresponsive genome of Daphnia pulex. Science 2011, 331(6017):555-561.

6. Grbic M, Van Leeuwen T, Clark RM, Rombauts S, Rouze P, Grbic V, Osborne EJ, Dermauw W, Thi Ngoc PC, Ortego F, et al: The genome of Tetranychus urticae reveals herbivorous pest adaptations. Nature 2011 479(7374):487-492

7. Gilbert D: OrthoMCL clustering among 14 arthropod proteins (ARP2). http:// arthropods.eugenes.org/arthropods, Dec. 2009, gilbertd@indiana.edu.
8. Clamp M, Fry B, Kamal M, Xie X, Cuff J, Lin MF, Kellis M, Lindblad-Toh K, Lander ES: Distinguishing protein-coding and noncoding genes in the human genome. Proc Natl Acad Sci USA 2007, 104(49):19428-19433.

9. Schmid K, Aquadro C: The evolutionary analysis of 'orphans' from the Drosophila genome identifies rapidly diverging and incorrectly annotated genes. Genetics 2001, 159:589-598.

10. Toll-Riera M, Bosch N, Bellora N, Castelo R, Armengol L, Estivill Z, Alba MM: Origin of primate orphan genes: a comparative genomic approach. Mol Biol Evol 2009, 26(3):603-612.

11. Das S, Yu L, Gaitatzes C, Rogers R, Freeman J, Blenkowska J, Adams R, Smith T, Lindelien J: Biology's new Rosetta Stone. Nature 1997, 385:29-30.

12. Skovgaard $M$, Jensen $L$, Brunak S, Ussery D, Krogh A: On the total number of genes and their length distribution in complete microbial genomes. Trends Genet 2001, 17(8):425-428.

13. Fukuchi S, Nishikawa K: Estimation of the number of authentic orphan genes in bacterial genomes. DNA Res 2004, 11:219-231.

14. Nagaraj S, Gasser R, Ranganathan S: A hitchhiker's guide to expressed sequence tag (EST) analysis. Brief Bioinform 2006, 8(1):6-21.

15. Adams MD, Kelley JM, Gocayne JD, Dubnick M, Polymeropoulos MH, Xiao H, Merril CR, Wu A, Olde B, Moreno RF, et al: Complementary DNA sequencing: expressed sequence tags and human genome project. Science 1991, 252(5013):1651-1656.

16. Haas BJ, Volforsky N, Town C, Troukhan M, Alexandrov N, Feldmann K, Flavell R, White O, Salzberg SL: Full-lenght messenger RNA sequences greatly improve genome annotation. Genome Biol 2002 3(6):research0029.0021-0029.0012.

17. Rudd S, Mewes H-W, Mayer K: Sputnik: a database platform for comparative plant genomics. Nucleic Acids Res 2003, 31(1):128-132.

18. Bonaldo M, Lennon G, Soares M: Normalization and subtraction: two approaches to facilitate gene discovery. Genome Res 1996, 6:791-806.

19. Hill C, Wikel S: The genome project: an opportunity for advancing tick research. Trends Parasitol 2005, 21(4):151-153.

20. Meyer JM, Kurtti TJ, Zee JP, Hill CA: Genome organization of major tandem repeats in the hard tick, Ixodes scapularis. Chromosome Res 2010, 18(3):357-370.

21. Guerrero FD, Moolhuijzen P, Peterson DG, Bidwell S, Caler E, Bellgard M, Nene VM, Djikeng A: Reassociation kinetics-based approach for partial genome sequencing of the cattle tick, Rhipicephalus (Boophilus) microplus. BMC Genomics 2010, 11:374.

22. Guerrero FD, Nene VM, George JE, Barker SC, Willadsen P: Sequencing a new target genome: the Boophilus microplus (Acari: Ixodidae) genome project. J Med Entomol 2006, 43(1):9-16.

23. Goodman J, Dennis D, Sonenshine DE: Tick-borne Diseases of Humans. Washington, D.C.: ASM Press; 2005.

24. Santos I, Valenzuela JG, Ribeiro JMC, De Castro M, Costa JN, Costa AM, Da Silva ER, Neto OBR, Rocha C, Daffre S, et al: Gene discovery in Boophilus microplus, the cattle tick - the transcriptomes of ovaries, salivary glands, and hemocytes vol. 1026. In Impact of Ecological Changes on Tropical Animal Health and Disease Control. Edited by Bokma BH, Blouin EF, Bechara GH; 2004:242-246.

25. Alarcon-Chaidez FJ, Sun J, Wikel SK: Transcriptome analysis of the salivary glands of Dermacentor andersoni Stiles (Acari: Ixodidae). Insect Biochem Mol Biol 2007, 37(1):48-71.

26. Batista IFC, Chudzinski-Tavassi AM, Faria F, Simons SM, Barros-Batestti DM, Labruna MB, Leao LI, Ho PL, Junqueira-de-Azevedo ILM: Expressed sequence tags (ESTs) from the salivary glands of the tick Amblyomma cajennense (Acari: Ixodidae). Toxicon 2008, 51(5):823-834.

27. Francischetti IMB, Mans BJ, Meng Z, Gudderra N, Veenstra TD, Pham VM Ribeiro JMC: An insight into the sialome of the soft tick, Ornithodorus parkeri. Insect Biochem Mol Biol 2008, 38(1):1-21.

28. Francischetti IMB, Pham VM, Mans BJ, Andersen JF, Mather TN, Lane RS, Ribeiro JMC: The transcriptome of the salivary glands of the female western black-legged tick Ixodes pacificus (Acari: Ixodidae). Insect Biochem Mol Biol 2005, 35(10):1142-1161.

29. Nakajima C, Vaz ID, Imamura S, Konnai S, Ohashi K, Onuma M: Random sequencing of cDNA library derived from partially-fed adult female Haemaphysalis longicornis salivary gland. J Vet Med Sci 2005, 67(11):1127-1131.

30. Nene V, Lee D, Quackenbush J, Skilton R, Mwaura S, Gardner MJ, Bishop R: $A v G l$, an index of genes transcribed in the salivary glands of the ixodid tick Amblyomma variegatum. Int J Parasitol 2002, 32(12):1447-1456. 
31. Valenzuela JG, Francischetti IMB, Pham VM, Garfield MK, Mather TN, Ribeiro JMC: Exploring the sialome of the tick Ixodes scapularis. J Exp Biol 2002, 205(18):2843-2864.

32. Aljamali MN, Hern L, Kupfer D, Downard S, So S, Roe BA, Sauer JR, Essenberg RC: Transcriptome analysis of the salivary glands of the female tick Amblyomma americanum (Acari: Ixodidae). Insect Mol Biol 2009, 18(2):129-154.

33. Chmelar J, Anderson JM, Mu J, Jochim RC, Valenzuela JG, Kopecky J: Insight into the sialome of the castor bean tick, Ixodes ricinus. BMC Genomics 2008, 9:233.

34. Francischetti IMB, Anderson JM, Manoukis N, Pham VM, Ribeiro JMC: An insight into the sialotranscriptome and proteome of the coarse bontlegged tick, Hyalomma marginatum rufipes. J Proteomics 2011, 74(12):2892-2908.

35. Francischetti IMB, Meng Z, Mans BJ, Gudderra N, Hall M, Veenstra TD, Pham VM, Kotsyfakis M, Ribeiro JMC: An insight into the salivary transcriptome and proteome of the soft tick and vector of epizootic bovine abortion, Ornithodoros coriaceus. J Proteomics 2008, 71(5):493-512.

36. Allan B, Dutra H, Goessling L, Barnett K, Chase J, Maquis R, Pang G, Storch G, Thach R, Orrock J: Invasive honeysuckle eradication reduces tick-borne disease risk by altering host dynamics. Proc Natl Acad Sci USA 2010, 107(43):18523-18527.

37. Childs J, Paddock C: The ascendancy of Amblyomma americanum as a vector of pathogens affecting humans in the United States. Ann Rev Entomol 2003, 48:307-337.

38. Paddock C, Yabsley J: Ecological havoc, the rise of white-tailed deer, and the emergence of Amblyomma americanum-associated zoonoses in the United States. Curr Top Microbiol 2007, 315:289-324.

39. Anderson BE, Dawson JE, Jones DC, Wilson KH: Ehrlichia chaffeensis, a new species associated with human ehrlichiosis. J Clin Microbiol 1991, 29(12):2838-2842

40. Armstrong PM, Brunet LR, Spielman A, Telford SR 3rd: Risk of Lyme disease: perceptions of residents of a Lone Star tick-infested community. Bull World Health Organ 2001, 79(10):916-925.

41. James AM, Liveris D, Wormser GP, Schwartz I, Montecalvo MA, Johnson BJ: Borrelia lonestari infection after a bite by an Amblyomma americanum tick. J Infect Dis 2001, 183(12):1810-1814.

42. Hopla, Downs C: The isolation of Bacterium tularense from the tick, Amblyomma americanum. J Kans Entomol Soc 1953, 26:71-72.

43. Parola P, Raoult D: Ticks and tickborne bacterial diseases in humans: an emerging infectious threat. Clinical Inf Dis 2001, 32:897-928.

44. McMullan LK, Folk SM, Kelly AJ, MacNeil A, Goldsmith CS, Metcalfe MG, Batten BC, Albariño CG, Zaki SR, Rollin PE, et al: A new phlebovirus associated with severe febrile illness in Missouri. N Engl J Med 2012, 367(9):834-841.

45. Benson D, Karsch-Mizrachi I, Lipman D, Sayers E: GenBank. Nucleic Acids Res 2011, 39:D32-37.

46. Aljamali MN, Ramakrishnan VG, Weng H, Tucker JS, Sauer JR, Essenberg RC: Microarray analysis of gene expression changes in feeding female and male lone star ticks, Amblyomma americanum (L). Arch Insect Biochem Physiol 2009, 71(4):236-253.

47. Tang Z, Choi J-H, Hemmerich C, Sarangi A, Colbourne J, Dong Q: ESTPiper - a web-based analysis pipeline for expressed sequence tags. BMC Genomics 2009, 10(1):174.

48. Huang $X$, Madan A: CAP3: a DNA sequence assembly program. Genome Res 1999, 9:868-877.

49. Consortium U: Reorganizing the protein space at the Universal Protein Resource (UniProt). Nucleic Acids Res 2012, 40:D71-D75.

50. Dujon B: The yeast genome project: what did we learn? Trends Genet 1996, 12(7):263-270.

51. Domazet-Loso T, Tautz D: An evolutionary analysis of orphan genes in Drosophila. Genome Res 2003, 13:2213-2219.

52. Fischer D, Eisenberg D: Finding families for genomic ORFans. Bioinformatics 1999, 15(9):759-762.

53. Alimi J, Poirot O, Lopez F, Claverie J: Reverse transcriptase-polymerase chain reaction validation of 25 "orphan" genes from Escherichia coli K-12 MG1655. Genome Res 2000, 10:959-966.

54. Jensen $\sqcup$, Skovgaard M, Sicheritz-Ponten T, Jorgensen MK, Lundegaard C, Pedersen CC, Petersen N, Ussery D: Analysis of two large functionally uncharacterized regions in the Methanopyrus kandleri AV19 genome. BMC Genomics 2003, 4(1):12.

55. Zdobnov EM, von Mering C, Letunic I, Torrents D, Suyama M, Copley RR, Christophides GK, Thomasova D, Holt RA, Subramanian GM, et al: Comparative genome and proteome analysis of Anopheles gambiae and Drosophila melanogaster. Science 2002, 298(5591):149-159.
56. Frith MC, Wilming LG, Forrest A, Kawaji H, Tan SL, Wahlestedt C, Bajic VB, Kai $C$, Kawai J, Carninci $P$, et al: Pseudo-messenger RNA: phantoms of the transcriptome. PLoS Genet 2006, 2(4):e23.

57. Shemesh R, Novik A, Edelheit S, Sorek R: Genomic fossils as a snapshot of the human transcriptome. Proc Natl Acad Sci USA 2006, 103(5):1364-1369.

58. Jeyaprakash A, Hoy MA: First divergence time estimate of spiders, scorpions, mites and ticks (subphylum: Chelicerata) inferred from mitochondrial phylogeny. Exp Appl Acarol 2009, 47(1):1-18.

59. Klompen JSH, Black WC, Keirans JE, Oliver JH: Evolution of ticks. Annu Rev Entomol 1996, 41:141-161.

60. Geraci NS, Johnston JS, Robinson JP, Wikel SK, Hill CA: Variation in genome size of argasid and ixodid ticks. Insect Biochem Mol Biol 2007, 37(5):399-408.

61. Ullmann A, Lima C, Guerrero F, Piesman J, Black WC IV: Genome size and organization in the blacklegged tick, Ixodes scapularis and the Southern cattle tick, Boophilus microplus. Insect Mol Biol 2005, 14(2):217-222

62. Hill CA, Tick and Mite Genomics Consortium: Genome analysis of major tick and mite vectors of human pathogens. 2010. White Paper.

63. Wang M, Guerrero FD, Pertea G, Nene VM: Global comparative analysis of ESTs from the southern cattle tick, Rhipicephalus (Boophilus) microplus. BMC Genomics 2007, 8:368.

64. Anderson JM, Sonenshine DE, Valenzuela JG: Exploring the mialome of ticks: an annotated catalogue of midgut transcripts from the hard tick, Dermacentor variabilis (Acari: Ixodidae). BMC Genomics 2008, 9:552.

65. Beaufays J, Adam B, Decrem Y, Prévôt P-P, Santini S, Brasseur R, Brossard M, Lins L, Vanhamme L, Godfroid E: Ixodes ricinus tick lipocalins: identification, cloning, phylogenetic analysis and biochemical characterization. PLoS One 2008, 3(12):e3941.

66. Nene V, Lee D, Kang'a S, Skilton R, Shah T, de Villiers E, Mwaura S, Taylor D, Quackenbush J, Bishop R: Genes transcribed in the salivary glands of female Rhipicephalus appendiculatus ticks infected with Theileria parva. Insect Biochem Mol Biol 2004, 34(10):1117-1128.

67. Lehane M: The Biology of Blood-Sucking in Insects. New York: Cambridge University Press; 2005.

68. Paterson S, Vogwill T, Buckling A, Benmayor R, Spiers AJ, Thomson NR, Quail M, Smith F, Walker D, Libberton B, et al: Antagonistic coevolution accelerates molecular evolution. Nature 2010, 464(7286):275-278.

69. Bishop JG, Dean AM, Mitchell-Olds T: Rapid evolution in plant chitinases: molecular targets of selection in plant-pathogen coevolution. Proc Natl Acad Sci USA 2000, 97(10):5322-5327.

70. Chou $\mathrm{H}-\mathrm{H}$, Holmes ME: DNA sequence quality trimming and vector removal. Bioinformatics 2001, 17(12):1093-1104.

71. Gauthier J-P, Legeai F, Zasadzinski A, Rispe C, Tagu D: AphidBase: a database for aphid genomic resources. Bioinformatics 2007, 23(6):783-784.

72. Lawson D: VectorBase: a data resource for invertebrate vector genomics. Nucleic Acids Res 2009, 37:D583-587.

73. Munoz-Torres M, Reese J, Childers C, Bennett A, Sundaram J, Childs K, Anzola J, Milshina N, Elsik C: Hymenoptera genome database: integrated community resources for insect species of the order Hymenoptera. Nucleic Acids Res 2011, 39:D658-D662

74. Wang J, Xia Q, He X, Dai M, Ruan J, Chen J, Yu G, Yuan H, Hu Y, Li R, et al: SilkDB: a knowledgebase for silkworm biology and genomics. Nucleic Acids Res 2005, 33:D399-D402.

75. Tweedie S, Ashburner M, Falls K, Leyland P, McQuilton P, Marygold S, Millburn G, Osumi-Sutherland D, Schroeder A, Seal R, et al: FlyBase: enhancing Drosophila Gene Ontology annotations. Nucleic Acids Res 2009, 37:D555-D559.

76. Wang L, Wang S, Li Y, Paradesi M, Brown S: Beetlebase: the model organism database for Tribolium castaneum. Nucleic Acids Res 2007, 35: D476-D479

77. Gilbert D, Singan V, Colbourne JK: wFleaBase: the Daphnia genomics information system. BMC Bioinformatics 2005, 6:45.

78. Min X, Butler G, Storms R, Tsang A: OrfPredictor: predicting protein-coding regions in EST-derived sequences. Nucleic Acids Res 2005, Web Server Issue:W677-W680.

79. Integrative Services for Genomic Analysis. http://isga.cgb.indiana.edu/Home. Indiana University Center for Genomics and Bioinformatics.

80. Drummond A, Ashton B, Buxton S, Cheung M, Cooper A, Duran C, Field M, Heled J, Kearse M, Markowitz S, et al: Geneious v5.4. 2011. Available from http://www.geneious.com/. 
81. Singh-Gasson S, Green RD, Yue Y, Nelson C, Blattner F, Sussman MR, Cerrina F: Maskless fabrication of light-directed oligonucleotide microarrays using a digital micromirror array. Nat Biotech 1999, 17(10):974-978.

82. Lopez JA, Colbourne JK: Dual-labeled expression-tiling microarray protocol for empirical annotation of genome sequences. CGB Technical Report. 2011.

83. Peterson M, Whittaker D, Ambreth S, Sureshchandra S, Buechlein A, Podicheti R, Choi J-H, Lai Z, Mockatis K, Colbourne J, et al: De novo transcriptome sequencing in a songbird, the dark-eyed junco (Junco hyemalis): genomic tools for an ecological model system. BMC Genomics 2012, 13(1):305.

84. Smyth G: Limma: linear models for microarray data. In Bioinformatics and Computational Biology Solutions using $R$ and Bioconductor. Edited by Gentleman R, Carey V, Dudoit S, Irizarry R, Huber W. New York: Springer; 2005:397-420.

85. Development Core Team R: R: A Language and Environment for Statistical Computing. Vienna, Austria: R Foundation for Statistical Computing; 2010.

doi:10.1186/1471-2164-14-135

Cite this article as: Gibson et al: Why so many unknown genes?

Partitioning orphans from a representative transcriptome of the lone star tick Amblyomma americanum. BMC Genomics 2013 14:135.

\section{Submit your next manuscript to BioMed Central and take full advantage of:}

- Convenient online submission

- Thorough peer review

- No space constraints or color figure charges

- Immediate publication on acceptance

- Inclusion in PubMed, CAS, Scopus and Google Scholar

- Research which is freely available for redistribution 